\title{
Reconstruction of the Three-Dimensional Mandibulofacial Defects Using a Single Cutaneous Perforator-Based Fibula Osteocutaneous Flap
}

\author{
In Sook Kang, \\ Jun Gul Ko, \\ JiSeon Choi, \\ Jin Soo Lim, \\ Min Cheol Kim \\ Department of Plastic and Reconstructive \\ Surgery, St. Vincent's Hospital, College of \\ Medicine, The Catholic University of Korea, \\ Seoul, Korea \\ No potential conflict of interest relevant to \\ this article was reported.
}

\begin{abstract}
The reconstruction of the mandibulofacial defects is a difficult task when there are fullthickness cheek defects involving mandible, inner mucosa and outer skin. There are several reconstructive options for the coverage of large defects, but most of the methods are complicated, and time- and effort-consuming. We hereby present a case of fibula osteocutaneous flap based on a single peroneal artery perforator in the reconstruction of a threedimensional mandibulofacial defects.
\end{abstract}

Keywords: Reconstruction / Microsurgery / Free flap / Perforator flap

\section{INTRODUCTION}

In the presence of full-thickness defects involving bony mandible, inner mucosa, and outer skin, the reconstruction of the mandibulofacial defects becomes complicated. First, it is difficult to make a three-dimensional shape of the bone flap with two layers, inner mucosa, and outer skin that would be similar to the anatomical structure, which would lead to a good aesthetic outcome. Furthermore, mandible serves several important function, such as, among others, mastication and speech. In this respect, for the reconstruction of mandibulofacial defects, it is important to fill the tissue with good-quality vascular pedicle [1].

There are several reconstructive options for the coverage of large defects, including the use of multiple free flaps, combination flaps, two skin paddles with independent perforators, as well as

\section{Correspondence: Min Cheol Kim}

Department of Plastic and Reconstructive Surgery, St. Vincent's Hospital, College of Medicine, The Catholic University of Korea, 93 Jungbu-daero, Paldal-gu, Suwon 16247, Korea

E-mail: wisethoth@hotmail.com

Received May 31, 2017 / Revised September 15, 2017 / Accepted September 15, 2017 prelamination and prefabrication techniques [2]. Therefore, for the effective reconstruction of wide and composite mandibulofacial defects, a complicated, time- and effort-consuming method should be chosen in numerous cases. At present, the most popular method used to reconstruct this complex defect is the vascularized fibula free flap.

In this study, we present a case of fibula osteocutaneous flap based on a single peroneal artery perforator in the reconstruction of three-dimensional mandibulofacial defects.

\section{CASE REPORT}

A 68-year-old male presented with the right buccal abscess and oral cutaneous fistula. The obtained biopsy results confirmed buccal squamous cell carcinoma and the patient received preoperative chemotherapy (Fig. 1). Surgical procedures of the right modified radical neck dissection and segmental mandibulectomy were subsequently performed, resulting in three-dimensional defects involving outer skin, inner mucosa, and bony structure (Fig. 
2A). The authors harvested a fibula osteocutaneous flap based on a single septocutaneous perforator and resurfaced the full-thickness defects including bone, inner mucosa, and outer skin.

After drawing a line connecting the most prominent points of the fibula head and lateral malleolus, the center of skin paddle was designed to be positioned approximately $3 \mathrm{~cm}$ behind the line. We tried to include as many perforator vessels as possible in the skin paddle, but there were limitations as confirmed by preoperative computed tomography (CT) and Doppler (Fig. 2B). Through the anterior incision, the peroneal muscle was elevated from the fibula

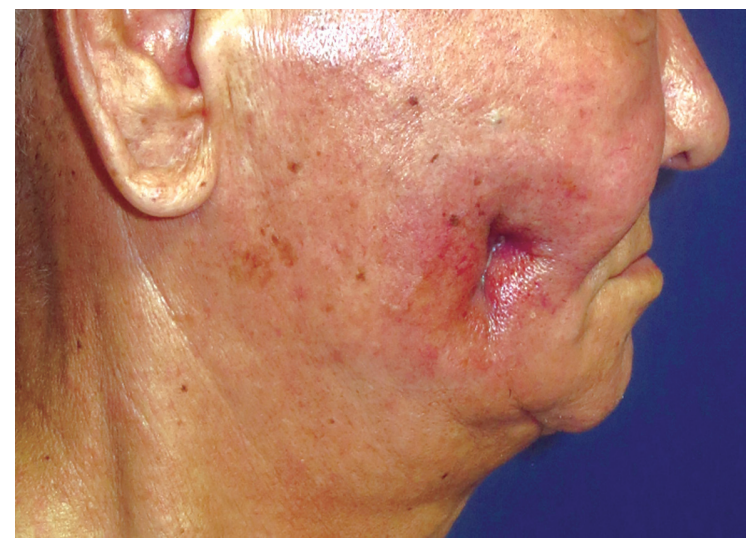

Fig. 1. Clinical presentation; oral-buccal fistula and ulceration on the right buccal area.
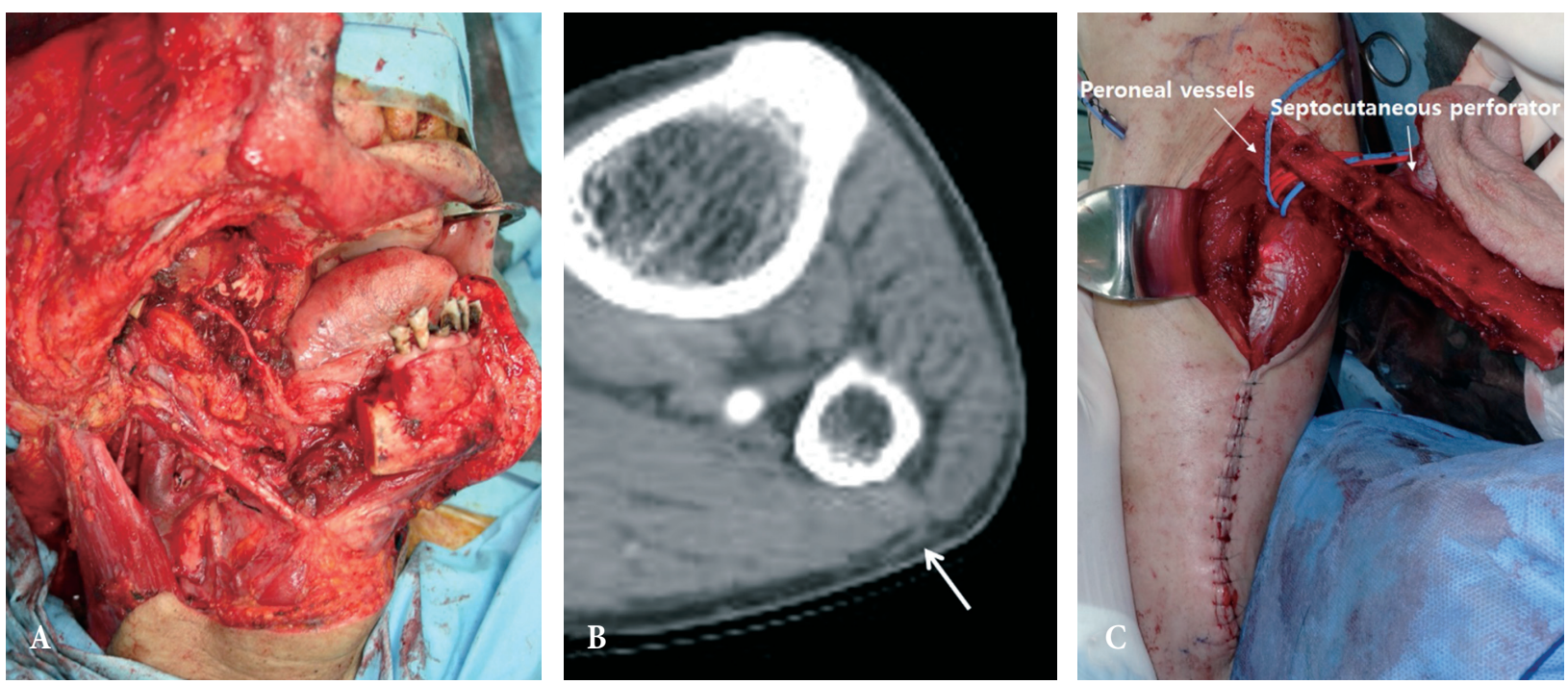

Fig. 2. (A) Three-dimensional defects including inner mucosa, bone and outer skin formed after mandibulectomy. (B) Computed tomography angiography. The arrow is indicating the septocutaneous perforator of the peroneal artery. (C) Havested fibula osteocutaneous flap based on a single perforator. 

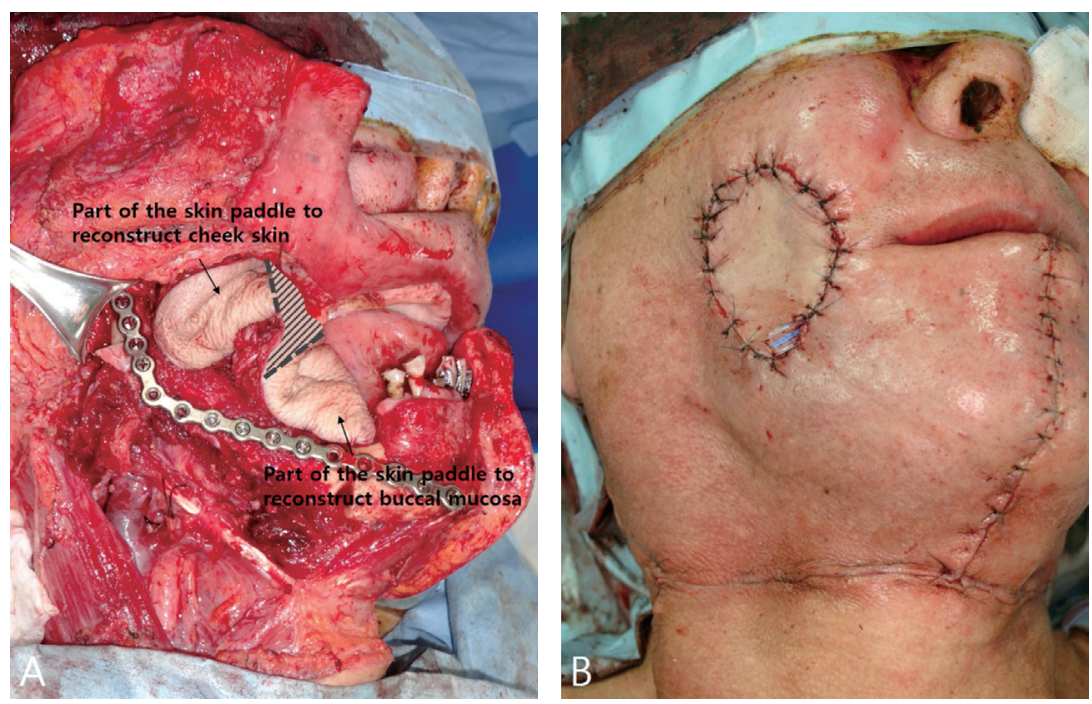

Fig. 3. (A) Intraoperative flap appearance after insetting. The hatched area refers to the part to be deepithelialized. (B) After fibula osteocutaneous flap reconstruction of the mandibulofacial defects.

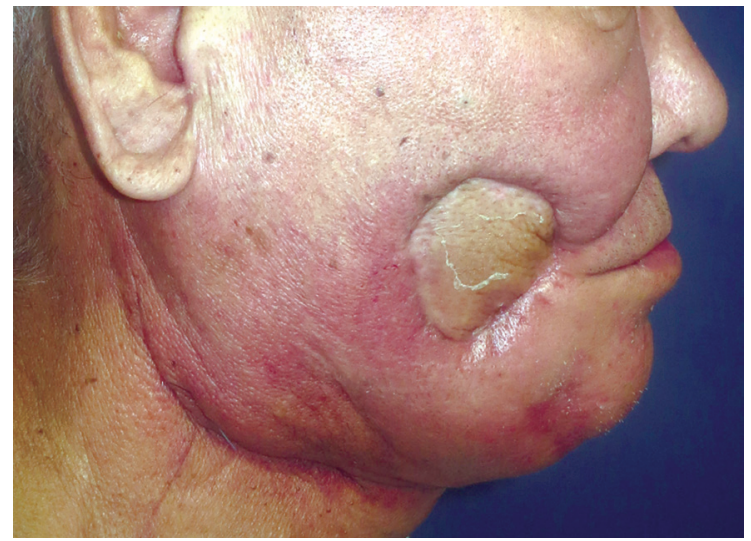

Fig. 4. Postoperative results 6 weeks following reconstruction.

significant complications. To date, 6 weeks after the surgery, the flap remains well-incorporated (Fig. 4).

\section{DISCUSSION}

The free fibular flap with microvascular anastomosis, first described by Taylor et al. [3] in 1974 and popularized by Hidalgo [4] in 1989, has become the first option for the mandibular reconstruction.

The fibular flap could offers a good length of the dense cortical bone, as well as sufficient height to match the height of the mandible through the 'double-barreling' technique of the fibular, leading to better aesthetical results. In addition, it provides pedicle based on the peroneal artery with a suitable diameter and has a low risk of bleeding and infection. Furthermore, low donor-site morbidity and the ability to have two team approaches simultaneously without the intraoperative position change are among the major advantages of the fibular flap for the reconstruction of mandible [1].

However, despite the advantages in the reconstruction of bony structure, the major drawback of the method is in the poor quality of its soft tissue component [5]. Accordingly, numerous papers have been published proposing various techniques to make up for this weakness. These include multiple free flap transfer or single free flap with two separate skin paddles [5]. Furthermore, single free flap with double skin paddle completely divided or de-epithelialized based on two different skin perforators was reported to reconstruct such composite defects [6]. A recent report showed a three-dimensional printing technique to obtain digital models and design the reconstructed mandible prior to the surgical procedure [7] and virtual surgical planning based on the CT and computer-aided design [8]. In the same vein, there have been numerous efforts to reduce the complications, including, among 
others, operative time and donor-site morbidity, especially with regard to insetting of the flap. However, due to being cost-intensive, these techniques are not available in all medical centers.

Peroneal vessels are usually branched from the posterior tibial artery and run to the foot along the posteromedial border of the fibula, responsible for the blood supply to the fibula, and located between the tibialis posterior muscle and the flexor halluces longus, with a variety of nutrient vessels and musculocutaneous branches. Several cutaneous vessels of peroneal vessels are located at the junctions of the upper and middle thirds of the fibula and are located between 15 and $27 \mathrm{~cm}$ of the fibula head. They are preserved during use of the skin paddle during surgery. The variation of popliteal branching is known, and caution is needed, especially in posterior surface of the fragile septum dissection, and there is a great risk of multiple osteotomies of the fibula as well as the septal perforator. Doppler and CT are widely used to detect this perforator, but we are not absolutely reliable because we encounter many risks during the operation.

In our case, we elevated the single free flap of a sufficient size to cover the defects based on a single perforator, separated the skin paddle by de-epithelialization of an intervening strip of skin, and covered the full-thickness defects of the mandibulofacial area. Further studies are warranted to determine whether or not this fibula osteocutaneous flap with a single perforator is on a par with the outcomes of more complicated and more cost- and time-consuming methods. Overall, the proposed method can reasonably be expected to enable a shorter operative time and a simpler operation without the use of expensive high technology. Also, its use would enable reconstructive surgeons to avoid making extra useless effort.

Therefore, we contend that this technique as a good alternative in cases when large, noncontiguous defects cannot be covered by simple free flaps.

\section{PATIENT CONSENT}

The patient provided written informed consent for the publication and the use of their images.

\section{REFERENCES}

1. Kokosis G, Schmitz R, Powers DB, Erdmann D. Mandibular reconstruction using the free vascularized fibula graft: an overview of different modifications. Arch Plast Surg 2016;43:3-9.

2. Torroni A, Marianetti TM, Romandini M, Gasparini G, Cervelli D, Pelo S. Mandibular reconstruction with different techniques. J Craniofac Surg 2015;26:885-90.

3. Taylor GI, Miller GD, Ham FJ. The free vascularized bone graft: a clinical extension of microvascular techniques. Plast Reconstr Surg 1975;55:533-44.

4. Hidalgo DA. Fibula free flap: a new method of mandible reconstruction. Plast Reconstr Surg 1989;84:71-9.

5. Leclere FM, Bosc R, Temam S, Leymarie N, Mirghani H, Sarfati B, et al. Reconstruction of large mandibulofacial defects with the composed double skin paddle fibula free flap: a review of 32 procedures. Laryngoscope 2014;124:1336-43.

6. Kubo T, Osaki Y, Hattori R, Kanazawa S, Hosokawa K. Reconstruction of through-and-through oromandibular defects by the doubleskin paddle fibula osteocutaneous flap: can the skin paddle always be divided? J Plast Surg Hand Surg 2013;47:46-9.

7. Tsai MJ, Wu CT. Study of mandible reconstruction using a fibula flap with application of additive manufacturing technology. Biomed Eng Online 2014;13:57.

8. Monaco C, Stranix JT, Avraham T, Brecht L, Saadeh PB, Hirsch D, et al. Evolution of surgical techniques for mandibular reconstruction using free fibula flaps: the next generation. Head Neck 2016;38 Suppl 1:E2066-73. 